Provided for non-commercial research and education use. Not for reproduction, distribution or commercial use.

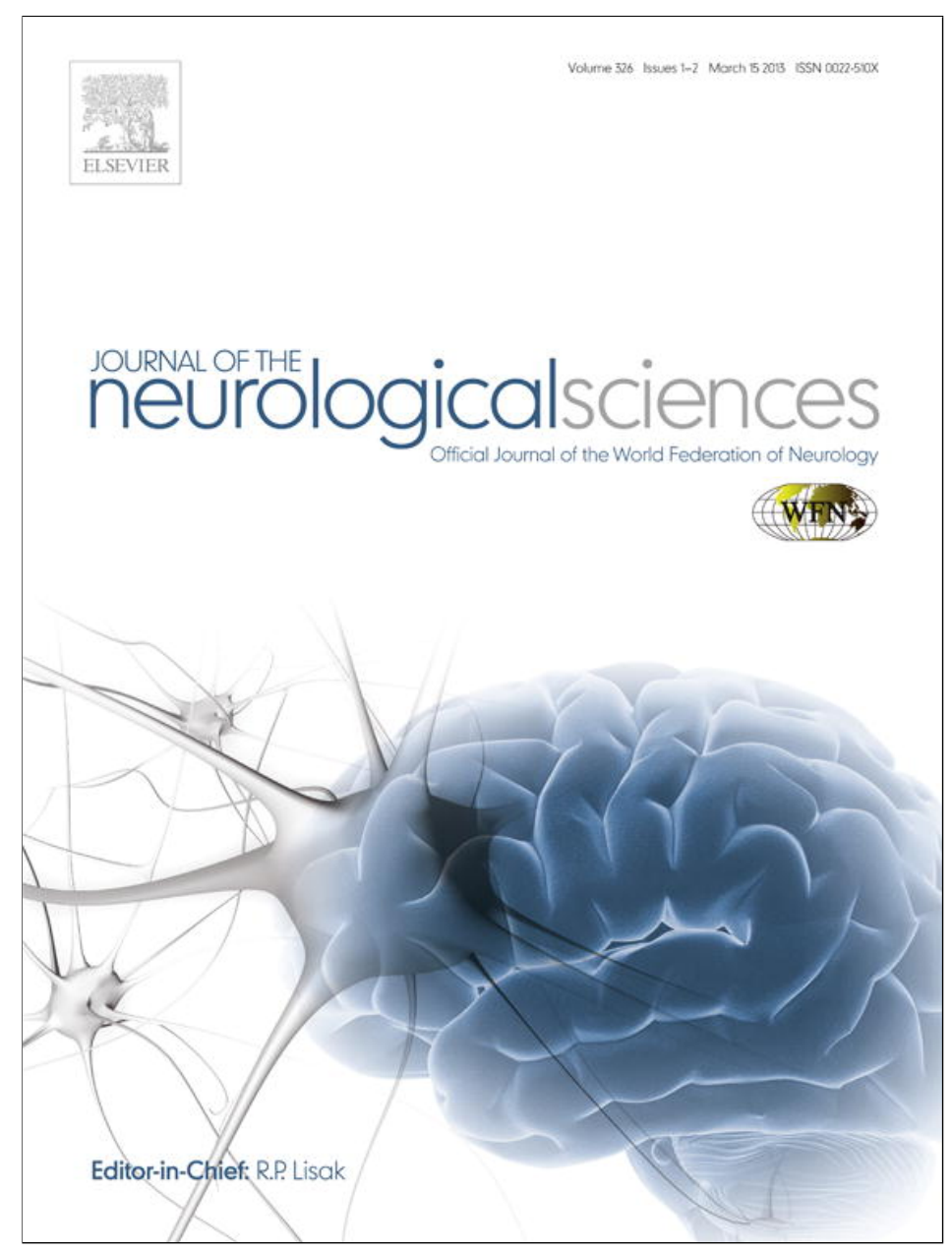

This article appeared in a journal published by Elsevier. The attached copy is furnished to the author for internal non-commercial research and education use, including for instruction at the authors institution and sharing with colleagues.

Other uses, including reproduction and distribution, or selling or licensing copies, or posting to personal, institutional or third party websites are prohibited.

In most cases authors are permitted to post their version of the article (e.g. in Word or Tex form) to their personal website or institutional repository. Authors requiring further information regarding Elsevier's archiving and manuscript policies are encouraged to visit:

http://www.elsevier.com/copyright 
Short communication

\title{
Sporadic Parkinson disease and Amyotrophic Lateral Sclerosis complex (Brait-Fahn-Schwartz Disease)
}

\author{
Concetta Manno, Alessio Lipari, Valeria Bono, Alfonsa Claudia Taiello, Vincenzo La Bella* \\ ALS Clinical Research Center, Department of Experimental Biomedicine and Clinical Neurosciences, University of Palermo, Italy
}

\section{A R T I C L E I N F O}

\section{Article history:}

Received 23 November 2012

Received in revised form 6 January 2013

Accepted 8 January 2013

Available online 4 February 2013

\section{Keywords:}

Parkinsonism

Parkinson's disease

Amyotrophic Lateral Sclerosis

PD-ALS complex

\begin{abstract}
A B S T R A C T
Clinical evidence for parkinsonism may accompany Amyotrophic Lateral Sclerosis with a frequency ranging from 5\% to 17\%. The concurrence of Amyotrophic Lateral Sclerosis and Parkinson's disease, outside the known Guam and Kii Peninsula foci, is instead rare, but this raises the possibility of a common pathogenesis. Clinically this complex presents with a levodopa-responsive parkinsonism and Amyotrophic Lateral Sclerosis and has been termed Brait-Fahn-Schwartz disease.

Here we describe two patients with this uncommon neurodegenerative complex. Both presented with Parkinson disease and progressed to a full blown Amyotrophic Lateral Sclerosis. We further suggest that the association of Parkinson disease and Amyotrophic Lateral Sclerosis represents a distinct nosological entity, which should be kept separated from extrapyramidal signs and symptoms that may occur in Amyotrophic Lateral Sclerosis.
\end{abstract}

(c) 2013 Elsevier B.V. All rights reserved.

\section{Introduction}

Extrapyramidal signs and symptoms due to nigrostriatal dysfunction have been reported in patients with Amyotrophic Lateral Sclerosis (ALS) [1-5]. However, outside the known ALS-Parkinson-Dementia complex (ALS-PD) of the Guam and Kii Peninsula of Japan, the comorbidity of Parkinson's disease and ALS is rare. This uncommon neurodegenerative complex was first described by Brait et al. [6]. The Parkinson's diseaseALS complex (also termed with the eponym Brait-Fahn-Schwartz disease [4]) clinically presents itself with a levodopa-responsive parkinsonism followed by ALS, and can either be sporadic or familial [6-8].

Here, we report the clinical, laboratory and imaging studies of two patients with idiopathic Parkinson's disease and ALS, and discuss the nosologic and pathogenic implications of these two rarely overlapping neurodegenerative disorders.

\section{Case reports}

The first patient (A) was a 55 year-old woman with a six-month history of resting tremor and cogwheel rigidity of her right hand and arm and a moderate bradykinesia; her medical and genetic history was negative. Familial history for extrapyramidal disorders was also negative. The diagnostic workup was performed in another hospital

* Corresponding author at: ALS Clinical Research Center, Via G La Loggia 1, University of Palermo, 90129 Palermo, Italy. Tel.: + 390916555158.

E-mail address: vincenzo.labella@unipa.it (V. La Bella). and a diagnosis of Parkinson's disease (PD) was made, based on the clinical presentation and a good response to a levodopa load. Other causes of parkinsonism were excluded.

She started a therapy with pramipexole and levodopa, with a significant improvement in her extrapyramidal symptoms and signs. About one year after the diagnosis of PD, she began to complain of weakness in her right lower limb. A right foot drop appeared. She was referred to our Hospital. Neurological examination showed muscle atrophy and weakness with spastic rigidity in the lower limbs, more pronounced in the right leg. A moderate muscle atrophy was noted in both hands and arms. Fasciculations were clinically evident in the trunk and both upper limbs. Bilateral hyperactive deep tendon reflexes, Hoffman's and Babinski's signs were present.

Mini Mental State Examination (MMSE) scored 30/30. Extensive biochemical laboratory investigations were negative. Electromyography revealed a neurogenic pattern of denervation with fibrillations and positive sharp waves in the four limbs. MRI of the brain showed only a mild cortical atrophy while SPECT analysis with ${ }^{123}$ I-Ioflupane revealed a bilateral reduced $(\approx-45 \%$ ) striatal uptake (Fig. 1). Genetic screening for SOD1, TDP-43, C9orf 72, FUS, angiogenin, Park-1, Park-2, Park-6 and Park-7 (DJ-1) mutations was negative.

A diagnosis of ALS, according to El-Escorial-revised criteria [9], which involves degeneration of the upper and lower motor neurons, was made and riluzole was started. While the parkinsonian symptoms continued to be controlled by the therapy, muscle atrophy and weakness worsened and the patient six months later became wheel-chair bound. Respiratory insufficiency ensued two years after ALS onset. A non-invasive ventilation was started, but the patient died after few months because of acute respiratory failure. 

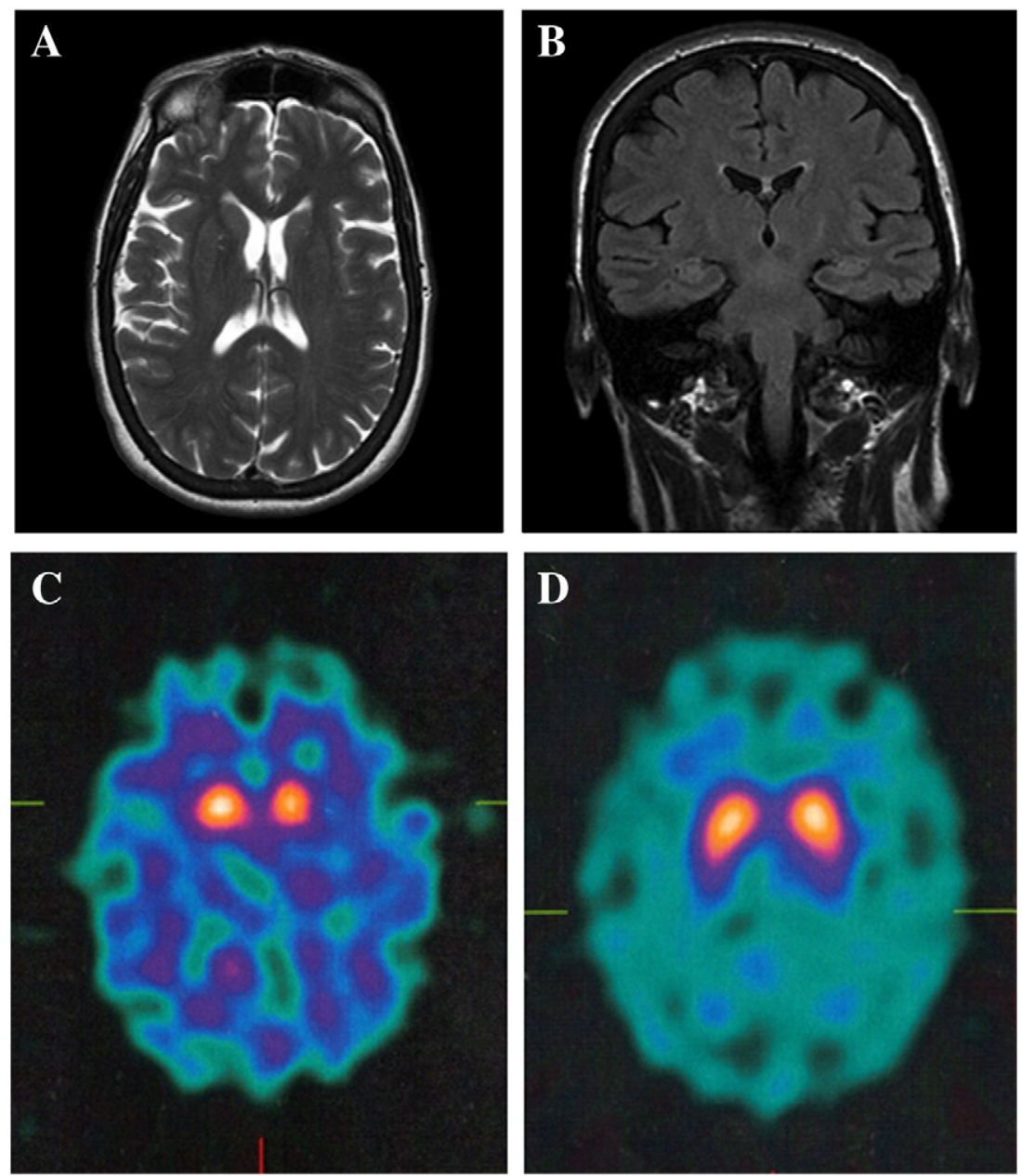

Fig. 1. MRI and ${ }^{123}$ I-Ioflupane SPECT of the patient A. Axial T2-weigthed (A) and FLAIR coronal (B) MRI show a mild cortical atrophy with no altered signals in the white matter. ${ }^{123}$ I-Ioflupane SPECT of the basal ganglia demonstrated a bilateral reduced striatal uptake, with a slight prevalence on the left side (C). A ${ }^{123}$ I-Ioflupane SPECT image of an unrelated age-matched control is shown for comparison (D).

The second patient (B) is a 69-year-old man with a two-year history of bradykinesia and resting tremor of his left hand, which progressed to the right hand. Familial history for extrapyramidal disorders was negative. He was diagnosed with PD in another hospital. The diagnostic workup allowed exclusion of other causes of parkinsonism. A therapy with low-doses of levodopa was started which led to a significant improvement of the bradikynesia and, to a less extent, of the resting tremor. Sixteen months later, weakness and muscle atrophy of upper limbs appeared, which were more disabling in the left hand. As the patient is left-handed, this led to a marked difficulty in opening bottles, eating or dressing himself: as a parkinsonian patient he had never experienced those symptoms. During the following months, weakness and muscle atrophy worsened and fasciculations in the upper limb were noted. He was thus referred to our Center for evaluation.

The neurological examination the patient was hypomimic. Posture was slightly camptocormic with trunk rigidity and minimal shuffling gait. Moderate cogwheel rigidity and tremor (mixed, postural and resting) were present in the left hand. He showed a mild dysphagia and dysarthria. The tongue was atrophic with fasciculations. Weakness and muscle atrophy with frequent fasciculations were detected bilaterally in the shoulder girdles and upper limbs. Tendon reflexes were brisk in the upper limbs, with the presence of the Hoffmann sign. In the lower limbs only a very mild atrophy was evident with increased tendon reflexes and a moderate rigidity. Coordination and all sensory modalities were normal. MMSE scored 28/30. The biochemical workup, which included a cerebrospinal fluid analysis, was negative. EMG showed widespread denervation in the four limbs and tongue. Brain MRI of documented a mild cortical atrophy. SPECT analysis ${ }^{123}$ I-Ioflupane demonstrated a bilateral reduced $(-35 \%)$ striatal uptake. This patient underwent genetic screening for SOD1, TDP-43, C9orf72, FUS, angiogenin, Park-1, Park-2, Park-6, Park-7 (DJ-1) mutations which was negative. A diagnosis of Parkinson disease with clinically-probable ALS was made [9]. He continued the levodopa therapy and riluzole was added with a transient improvement of the resting tremor. The clinical course was however progressive and now the patient is wheel-chair bound and fed through a PEG device.

\section{Discussion}

We have presented two patients with an idiopathic Parkinson's disease (PD) who later, in the course of their disease, developed Amyotrophic Lateral Sclerosis. This neurodegenerative complex, indeed very rare, has been given the eponym of Brait-Fahn-Schwartz disease $[4,6]$. Growing evidence suggests that this association is in fact not coincidental, as it may share a common pathogenic basis $[4,7]$.

Parkinsonism may associate with motor neuron disease (MND) in various neurodegenerative conditions, such as multiple system atrophy, hereditary spastic paraplegia, SCAs, fronto-temporal lobar degeneration. In these disorders, MND usually manifests as amyotrophy, expression of lower motor neuron degeneration.

Symptoms and signs of parkinsonism have also been described in ALS $[2,4,6,10-12]$, with a frequency ranging from $5 \%$ to $17 \%$ [12-14]. 
ALS however is a distinct MND, which involves both upper and lower motor neurons-related symptoms and signs, and it shows a steadily clinical progression. When associated to ALS, parkinsonian signs and symptoms have been referred to as slowness of gait with postural instability, rigidity and bradykinesia with an overall poor response to levodopa therapy $[11,15]$. In a large series of ALS patients, 39 cases $(17 \%)$ with prevalent stiffness were identified in which extrapyramidal features of rigidity and postural instability were found to be highly correlated with the pyramidal signs [12]. This association has been recently challenged, as no correlation was found between clinical rating scales assessing bradykinesia and postural instability and the degree of nigrostriatal impairment on ${ }^{123}$ I-Ioflupane scintigraphy in patients with upper motor neuron dominant ALS [16].

There is evidence, however, of a decreased striatal dopaminergic function in ALS $[5,14,17]$. Furthermore, necropsy studies have shown neuronal loss in the substantia nigra and globus pallidus of ALS patients $[18,19]$. This strongly suggests that the extrapyramidal system is actually involved in this neurodegenerative disease, and this raises the question as to whether ALS should be included among the multisystem disorders.

A dominant axial rigidity with bradykinesia and a lack of pharmacological response to levodopa are the most striking features seen in ALS patients with parkinsonism $[4,11,15]$. Our two unrelated Brait-FahnShwartz disease (i.e., PD-ALS complex) patients were cognitively normal and showed clinical and imaging features consistent with a clinical diagnosis of PD, with a good response to dopaminergic therapy and a clear-cut clinical progression of the extrapyramidal symptoms before the appearance of the upper and lower motor neuron degeneration $[4,6]$. Recently, a PD-ALS syndrome with dementia has been described to be associated with mutations of DJ-1 (Park-7), a gene causing earlyonset PD [8]. This oncogene protein is widely expressed in CNS and it plays a neuroprotective role mitochondria-mediated against oxidative stress in PD and ALS models [20,21]. Thus, there is increasing evidence that PD and ALS may share a common pathogenesis in a subgroup of patients.

In conclusion, these two cases of Brait-Fahn-Schwartz disease reinforce the evidence that the association of PD and ALS might represent a distinct pathological entity $[4,8]$. This should be separated from the extrapyramidal signs and symptoms that may appear in ALS. The positive and persistent response to dopaminergic drug therapy and the sequence by which progressive parkinsonian symptoms and signs precede the appearance of ALS represent the two strong clinical markers of this rare syndrome.

\section{Conflict of interest}

The authors declare that they have no conflict of interest.

\section{Acknowledgments}

We thank Mr Martin Russell for the revision of the English language.

\section{References}

[1] Kato S, Oda M, Tanabe H. Diminution of dopaminergic neurons in the substantia nigra of sporadic amyotrophic lateral sclerosis. Neuropathol Appl Neurobiol 1993;19: 300-4.

[2] Desai J, Swash M. Extrapyramidal involvement in amyotrophic lateral sclerosis: backward falls and retropulsion. J Neurol Neurosurg Psychiatry 1999;67:214-6.

[3] Takahashi H, Snow BJ, Bhatt MH, Peppard R, Eisen A, CalneMackenzie IR, et al. Extrapyramidal features in patients with motor neuron disease and dementia. A clinicopathological correlative study. Acta Neuropathol 2004;107:336-40.

[4] Wolf Gilbert RM, Fahn S, Mitsumoto H, Rowland LP. Parkinsonism and motor neuron diseases: twenty-seven patients with diverse overlap syndromes. Mov Disord 2010;25:1868-75.

[5] Park HK, Lim YM, Kim JS, Lee MC, Kim SM, Kim BJ, et al. Nigrostriatal dysfunction in patients with amyotrophic lateral sclerosis and parkinsonism. J Neurol Sci 2011;301:12-3.

[6] Brait K, Fahn S, Schwarz GA. Sporadic and familial parkinsonism and motor neuron disease. Neurology 1973;23:990-1002.

[7] Lim Y-M, Park HK, Kim JS, Lee CS, Chung SJ, Kim J, et al. Clinical and neuroimaging characteristics in neurodegenerative overlap syndrome. Neurol Sci 2012, http://dx.doi.org/ 10.1007/s10072-012-1139-1.

[8] Annesi G, Savettieri G, Pugliese P, D'Amelio M, Tarantino P, Ragonese P, et al. DJ-1 mutations and parkinsonism-dementia-amyotrophic lateral sclerosis complex. Ann Neurol 2005;58:803-7.

[9] Brooks BR, Miller RG, Swash M, Munsat TL. El Escorial revisited: revised criteria for the diagnosis of amyotrophic lateral sclerosis. Amyotroph Lateral Scler 2000;1:293-9.

[10] Hasegawa K, Kowa H, Yagishita S. Extrapyramidal system involvement in motor neuron disease. J Neurol Sci 1992;108:137-48.

[11] Qureshi AI, Wilmot G, Dihenia B, Schneider JA, Krendel DA. Motor neuron disease with parkinsonism. Arch Neurol 1996;53:987-91.

[12] Pradat P-F, Bruneteau G, Munerati E, Salachas F, Le Forestier N, Lacomblez L, et al. Extrapyramidal stiffness in patients with amyotrophic lateral sclerosis. Mov Disord 2009;24:2143-8.

[13] Takahashi H, Snow BJ, Bhatt MH, Peppard R, Eisen A, Calne DB. Evidence for a dopaminergic deficit in sporadic amyotrophic lateral sclerosis on positron emission scanning. Lancet 1993;342:1016-8.

[14] Hideyama T, Momose T, Shimizu J, Tsuji S, Kwak S. A positron emission tomography study on the role of nigral lesions. Arch Neurol 2006;63:1719-22.

[15] Zoccolella S, Palagano G, Fraddosio A, Russo I, Ferramini E, Serlenga L, et al ALS-plus: 5 cases of concomitant amyotrophic lateral sclerosis and parkinsonism. Neurol Sci 2002;23:S123-4.

[16] D'Ascenzo C, Cecchin D, Santelli L, Palmieri A, Gaiani G, Cima V, et al Parkinson-like features in ALS with predominant upper motor neuron involvement. Amyotroph Lateral Scler 2012;13:137-43.

[17] Fathinia P, Hermann A, Reuner U, Kassubek J, Storch A, Ludolph AC. Parkinson's disease-like midbrain hyperechogenicity is frequent in amyotrophic lateral sclerosis. J Neurol 2012, http://dx.doi.org/10.1007/s00415-012-6654-8.

[18] Sasaki S, Tsutsumi Y, Yamane K, Sakuma H, Maruyama S. Sporadic amyotrophic lateral sclerosis with extensive neurological involvement. Acta Neuropathol 1992;84:211-5

[19] Yokota O, Tsuchiya K, Osa T, Ishihara T, deSilva R, Lees AJ, et al. Amyotrophic lateral sclerosis with dementia: an autopsy case showing many Bunina bodies, tau-positive neuronal and astrocytic plaque-like pathologies, and pallido-nigral degeneration. Acta Neuropathol 2006;112:633-45.

[20] Yamashita S, Mori A, Kimura N, Mita S, Maeda Y, Hirano T, et al. DJ-1 forms complexes with mutant SOD1 toxicity. J Neurochem 2010;113:860-70.

[21] Wang X, Petrie TG, Liu Y, Liu J, Fujikota H, Zhu X. Parkinson's disease-associated DJ-1 mutations impair mitochondrial dynamics and cause mitochondrial dysfunction. J Neurochem 2012;121:830-9. 